\title{
Application of negative pressure wound therapy in scalp reconstruction
}

\author{
Bohac $\mathrm{M}^{1}$, Mikusz $\mathrm{K}^{2}$, Fedeles $\mathrm{J} \mathrm{Sr}^{1}$ \\ Department of Plastic Surgery, University Hospital Bratislava, Faculty of Medicine, Comenius University \\ in Bratislava, Bratislava, Slovakia. bohac.md@gmail.com
}

\section{ABSTRACT}

Scalp defects in old polymorbid patients are still therapeutic challenge for reconstructive surgeons. We present the case of a male who underwent an excessive tumour resection with the unsuccessful skin graft coverage. The patient developed a progressive skin graft necrosis and infection with an exposure of calvarial bone. Initial surgical debridement and topical treatment resulted in an excessive bone exposure. We decided to use a negative pressure therapy after multiple bone trephinations, to improve growth of new vital tissue in bone-exposed area. This maneuverer, followed by a split thickness skin graft coverage, allowed a progressive defect healing. In such old polymorbid patient, calvarial bone trephinations with a negative pressure therapy could be considered to achieve effective and considerable results (Fig. 4, Ref. 16). Text in PDF www.elis.sk.

KEY WORDS: scalp reconstruction, negative pressure therapy, polymorbidity.

\section{Introduction}

We present our experience with the negative pressure wound therapy (NPWT) in patients with defects after previous tumours resection with a central exposure of calvarial bone. Different operative techniques can be applied for deep head wound: primary closure, skin graft and flaps: random, pedicled or free. The choice of the treatment depends on its size, depth, localization of the defect and the quality of the surrounding tissue (1). Due to the limited mobility of the scalp tissue, only small defects can be primarily closed. Skin grafting is possible, when the pericranium is present. Deep wound with denuded periosteum of calvarium or exposed dura requires a stable soft tissue coverage such as flaps (2). In the presented case, the plastic surgery procedures (flaps) could not be used for coverage of the calvarial bone, because of patient's poor health. Bone trephination in combination with NPWT was the treatment of choice for these wounds. The NPWT is widely used in the field of wound care $(3,4)$. The NPWT is a modified wound dressing, where a porous material (usually gauze or foam) is placed in the wound bed and enclosed using polyurethane films to form an airtight scalp. An external mechanical pump is connected to the dressing via tubing for its entire duration to provide suction and collect the wound aspirate (4). Deep scalp wounds with an exposed calvarial bone require a vital

${ }^{1}$ Department of Plastic Surgery, University Hospital Bratislava, Faculty of Medicine, Comenius University in Bratislava, Bratislava, Slovakia, and ${ }^{2}$ Department of Plastic Surgery, Centre for Burns Treatment, Siemianowice Slaskie, Poland

Address for correspondence: M. Bohac, MD, PhD, Department of Plastic Surgery, University Hospital Bratislava, Medical Faculty of Comenius University in Bratislava, Ruzinovska 6, SK-826 06 Bratislava, Slovakia. Phone: +421903316429 granulation tissue. The negative pressure therapy can successfully encourage granulation tissue formation. The use of the subatmospheric pressure stimulate or assist wound healing together with an induction of the new granulation tissue formation. Typically, the pump is set to continuously provide 80 to $125 \mathrm{mmHg}$ of a negative pressure. The NPWT allows to remove oedema/excess wound fluid, reduces gap between capillaries and wound cells, impacts improvement of oxygenation and nourishment of the wound. The NPWT removes some sloughed tissue, decreases bacterial proliferation and increases opportunity for proper granulation. The NPWT does not remove eschar. Surgical debridement of the wound must be performed at first. Improved blood supply can be caused by a reduction of oedema and enhancement of the wound perfusion. Negative and intermittent pressure reduces periwound hypo perfusion. The NPWT leads to stimulation of endothelial proliferation and angiogenesis. That brings blood supply back into the wound, what is required for delivery of cells, factors and elements (Platelets, Neutrophils, Monocytes, Nutrients, Oxygen). Peripheral blood supply is essential for a proper wound healing process $(4,5)$. The NPWT is indicated in patients with large soft tissue defects, in initially and postoperatively infected wounds after surgical debridement, and for skin graft fixation. The NPWT has been proved to be successful in treating soft tissue defects, where primary suture is unacceptable or impossible and in the wounds with an exposed bradytrophic tissue, such as bone or tendons $(3,6,7)$.

\section{Case report}

An 83-year-old polymorbid male underwent tumours resection in parieto-occipital region with an unsuccessful full thickness skin graft coverage and subsequent calvarial bone exposi- 


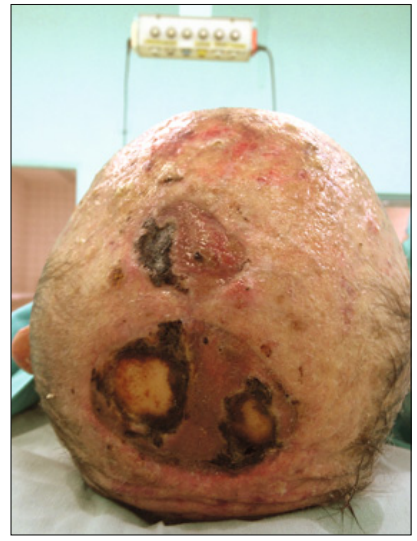

Fig. 1. The patients' first photo of the head before debridement in our department.

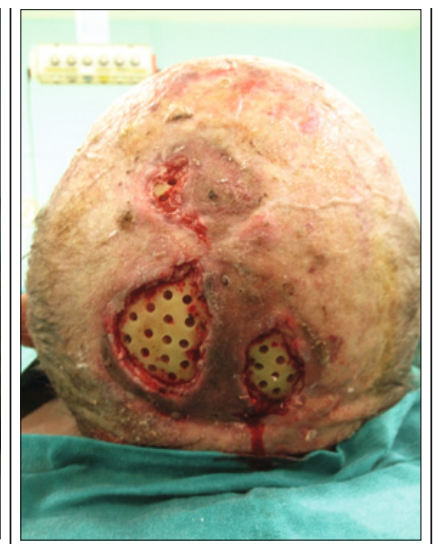

Fig. 2. Patient after debridement and initial trephination of the skull. Lack of bleeding from trephination is present.

tion. Histological examination revealed a complete extirpation of basocelular carcinoma. Three weeks after the initial operation, the patient arrived to our hospital and the cranial defects were evaluated. Complete dry necrotic skin grafts were situated in the parieto-occipital region at place of defects after previous tumours resection with central exposure of the calvarial bone (Fig. 1). He suffered from systemic necrotizing vasculitis with chronic renal failure on dialysis treatment, ischemic heart disease, arterial hypertension and secondary anaemia in his history. After the initial surgical debridement, the prophylactic antibiotic treatment with cefalexinum was started. The resultant soft tissue defect was together approximately $16 \mathrm{~cm}^{2}$. The first wound cultures showed colonization by Pseudomonas aeruginosa and Candida albicans without any systemic symptoms. According to the pharmacologist's recommendation, we started just local therapy with Acidum aceticum dressing. The wound was not suitable for complex flap reconstruction in this condition. According to the age and comorbidities of the patient, we decided to use NPT ( VivanoTec ${ }^{\mathrm{R}}$ Hartmann ) to promote granulation and to treat the infection before a definitive wound covering. We also performed a bone trephination for wound optimization. After calvarial trephination, a limited vitality, according to lack of bleeding, of the diploe was observed (Fig. 2). The NPT was activated on $125 \mathrm{mmHg}$ intermittent suction pressure. Dressings were changed in the isolation ward once every 4 days under strict aseptic conditions. After 4 weeks of the NPT, we achieved a significant improvement of the wound condition (Fig. 3), granulation tissue had covered all the exposed bone and the infection was successfully eradicated. The swelling had decreased, and the wound had contracted, leaving a total defect of $10 \mathrm{~cm}^{2}$. At this time, we decided to use a split-thickness skin graft in combination with the NPT for the definitive wound covering. Five days after the skin grafting procedure, a completely vital skin transplant was observed and the patient was discharged (Fig. 4). During 4 months of follow-up, no infectious or necrotic relapses occurred, and the patient is looking forward to relatively healthy life.

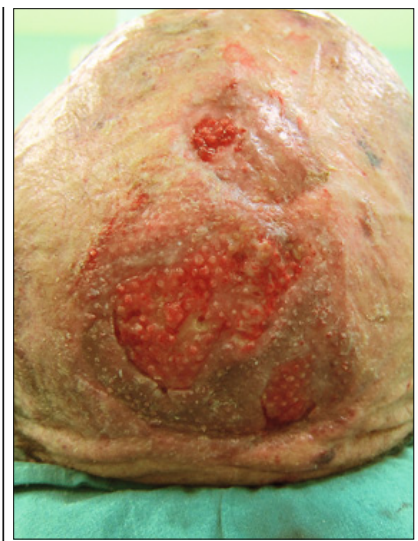

Fig. 3. The scalp with granulations after 4 weeks of application of the negative pressure therapy.

\section{Discussion}

The full thickness scalp defect can occur mainly after the removal of a malignant tumour on the scalp. In case of a malignant tumour on the scalp, wide excision is performed with a certain safety margin, which should include removal of the scalp in its full thickness. While tumour invasion on the pericranium is highly possible, the periosteum should also be removed. In this case, denuded skull is left exposed. The reconstruction of the deep head defects may only be obtained by using tissue with the same thickness and hair growth, so as to achieve an adequate coverage mobility and stability (8). The primary closure may be usually applied when the defect is $<3 \mathrm{~cm}^{2}$ to avoid tension, ischemia and necrosis, due to the limited elasticity of the scalp tissue. When the wound bed is well- vascularized and the size of wound is $>3 \mathrm{~cm}^{2}$, skin grafting is a suitable technique $(2,9)$. In case of a deep wound with a denuded periosteum, skin grafting is not the appropriate method. On the other hand, microdryling technique of lamina externa for granulation can delay the treatment with $3-5$ weeks. When the defect is between $3-25 \mathrm{~cm}^{2}$, the suitable reconstructive technique is the application of local flaps - random or pedicled $(9,10)$. The use of flaps is restricted by the ratio length/width. Local axial flap belongs to the fascio-cutaneous type and they can be planned in various shapes depending on the size, localization and the depth of the wound. These flaps provide solid, flexible and reliable tissue for large and deep wound. Considering the limited elasticity of the scalp, flaps must be planned slightly bigger than the wound. When the wound exceeds approximately $25 \mathrm{~cm}^{2}$ and surrounding tissue is damaged, the surgical options for reconstruction are the following: tissue expansion distal pedicled and free flaps. Free flaps employed for calvarial reconstruction includes: latissimus dorsi muscle flaps, antero-lateral thigh flap, radial forearm flaps, lateral arm flaps, rectus abdominis muscle flaps and scapular flaps (10). Tissue expansion is an effective method for expanding tissue and achieving an adequate coverage with the local flaps $(11,12)$. However, this technique is hard to perform in patients with severe defects and with badly damaged surrounding tissue. Planning and 
elevation of the distal pedicled flaps demand long operative hours and their relatively large size requires several surgical interventions to model and adapt them to the delicate structures of the head, the distal pedicle flaps are quite a good method of choice for covering big defects. The transfer of free flaps muscle or omental flaps is a safe and reliable technique for covering large and deep defects. This method is the preferred one in case of bone plate infection and a deficiency of local tissue $(12,13)$. When either the local or free flap is not recommended in such cases, it may be possible to perform a delayed two-stage split-thickness skin graft with various allograft materials. The reported cases in literature for scalp reconstruction with Matrix-derm or Integra followed by free skin graft or flap showed good functional and aesthetic results $(13,14,15)$. Unfortunately, the high price limits this application. When a flap is contraindicated, a large wound must heal by secondary intention and must be covered with the skin grafts. Healing by secondary intention is often slow in a patient for whom a flap is contraindicated. The skin graft requires a vascularized bed and will not take on bone. We performed an application of the negative pressure wound therapy on the trephined calve with subsequent split-thickness skin graft coverage, after cancer removal in patient with full thickness scalp defect accompanied with an exposure of the skull. The application of the negative pressure wound therapy improves the removal of wound exudate, provides a prevention against wound infection and shortens the time required for granulation tissue formation. It was difficult to perform a distal flap in this patient, because wound site had a poor vascular bed and the patient had poor systemic conditions. The reconstruction of wide scalp defect after a tumour removal is still a challenging problem in our surgical practice.

\section{References}

1. Lin SJ, Hanasono MM, Skoracki RJ. Scalp and Calvarial Reconstruction. Semin Plast Surg 2008; 22 (4): 281-293.

2. Zayakova Y, Stanev A, Mihailov H, Pashaliev N. Application of local axial flaps to scalp reconstruction. Arch Plast Surg 2013; 40 (5): 564-569.

3. Willy $\mathbf{C}$ et al. The theory and practice of vacuum therapy. Germany 2006 Chapter 1-22/ Clinical applications; 101-279.
4. Morykwas MJ, Argenta LC, Shelton-Brown EI, McGuirt W. Vacuum-assisted closure: a new method for wound control and treatment: animal studies and basic foundation. Ann Plast Surg 1997; 38 (6): 553-562.

5. Chen SZ, Li XY, Xu LS. Effects of vacuum assisted closure on wound microcirculation: an experimental study. Asian J Surg 2005; 28 (3): 211217.

6. Stannard JP, Volgas DA, Stewart R et al. Negative pressure wound therapy after severe open fractures: A prospective randomized study. J Orthop Trauma 2009; 23: 552-557.

7. DeFranzo AJ, Argenta LC, Marks MW et al. The use of vacuumassisted closure therapy for the treatment of lower extremity wounds with exposed bone. Plast Reconstr Surg 2001; 108: 1184-1191.

8. Marathe US, Sniezek JC. Use of the vacuum-assisted closure device in enhancing closure of a massive skull defect. Laryngoscope 2004; 114 (6): 961-964.

9. Leedy JE, Janis JE, Rohrich RJ. Reconstruction of acquired scalp defects: an algorithmic approach. Plast Reconstr Surg 2005; 116 (4): 54e-72e.

10. Chang KP. Free flap options for reconstruction of complicated scalp and calvarial defects: report of a series of cases and literature review. Microsurgery 2010; 30 (1): 13-18.

11. Wollina U, Bayyoud Y. Reconstruction of a large scalp defect by the sequential use of dermal substitute, self-filling osmotic tissue expander and rotational flap. J Cutan Aesthet Surg 2010; 3106-3110.

12. Ito $\mathrm{E}$, Watanabe $\mathrm{T}$, Sato $\mathrm{T}$ et al. Skull base reconstruction using various types of galeal flaps. Acta Neurochir 2012; 154: 179-185.

13. Mehrara BJ, Disa JJ, Pusic A. Scalp reconstruction. J Surg Oncol 2006; 94 (6): 504-508.

14. Corradino B, Di Lorenzo S, Barone AA et al. Reconstruction of full thickness scalp defects after tumour excision in elderly patients: Our experience with Integra dermal regeneration template. J Plast Reconstr Aesthet Surg 2010; 63: e245-e247.

15. Gonyon DL Jr, Zenn MR. Simple approach to the radiated scalp wound using Integra skin substitute. Ann Plast Surg 2003; 50: 315-320.

16. Momoh AO, Lypka MA, Echo A, Rizvi M, Klebuc M, Friedman JD. Reconstruction of full-thickness calvarial defect: a role for artificial dermis. Ann Plast Surg 2009; 62 (6): 656-659.

Received February 5, 2015. Accepted February 15, 2015. 